\title{
The reduced Ostrovsky equation: integrability and breaking
}

\author{
Roger Grimshaw \\ Department of Mathematical Sciences, \\ Loughborough University, U.K. \\ Karl Helfrich \\ Department of Physical Oceanography, \\ Woods Hole Oceanographic Institution, Woods Hole, U.S.A. \\ Ted Johnson \\ Department of Mathematics, \\ University College London, U.K. \\ May 10, 2012

\begin{abstract}
The reduced Ostrovsky equation is a modification of the Korteweg-de Vries equation, in which the usual linear dispersive term with a third-order derivative is replaced by a linear non-local integral term, which represents the effect of background rotation. This equation is integrable provided a certain curvature constraint is satisfied. We demonstrate, through theoretical analysis and numerical simulations, that when this curvature constraint is not satisfied at the initial time, then wave breaking inevitably occurs.
\end{abstract}

\section{Introduction}

It is well-known that the Korteweg-de Vries (KdV) equation can be used to model internal solitary waves in the atmosphere and ocean, see for instance the reviews by Grimshaw (2001) and Melville and Helfrich (2006),

$$
u_{t}+\mu u u_{x}+\lambda u_{x x x}=0 .
$$

Here $u(x, t)$ is the amplitude of an appropriate linear long wave mode, with linear long wave speed $c_{0}$, and (1) is expressed in a frame moving with that speed. The coefficients 
$\mu, \lambda$ are found from certain internal expressions involving the modal function and the background density stratification.

However, when the effects of background rotation through the Coriolis parameter $f$ need to be taken into account, an extra term is needed, and the KdV equation (1) is replaced by the Ostrovsky equation is, see Ostrovsky (1978), Grimshaw (1985) or the review by Grimshaw et al (1998),

$$
u_{t}+\mu u u_{x}+\lambda u_{x x x}=\gamma v, \quad v_{x}=u
$$

where $\gamma=f^{2} / 2 c_{0} \neq 0$. On the infinite line, the mass is zero

$$
\int_{-\infty}^{\infty} u d x=0, \text { and then also } \int_{-\infty}^{\infty} v d x=0 .
$$

For periodic solutions of period $2 L$, replace $(-\infty, \infty)$ with $(-L, L)$.

Our concern here is with the reduced Ostrovsky equation which is obtained by setting $\lambda=0$ in $(2)$,

$$
u_{t}+\mu u u_{x}=\gamma v, \quad v_{x}=u .
$$

Importantly, we note that when $\gamma=0$, equation (4) reduces to the inviscid Burgers (or Hopf) equation. It is then well-known and easily demonstrated that all localized solutions, or all periodic solutions, will break. That is the solution will develop an infinite slope in finite time. The issue then is how this breaking is affected when $\gamma \neq 0$. This is to be contrasted with the regularisation of the Hopf equation by the KdV equation (1) when it is known that breaking is replaced by the emergence of internal solitary waves, often in the form of internal undular bores.

The reduced Ostrovsky equation (4) (also known variously as the Ostrovsky-Hunter equation, or the Vakhnenko equation) has been previously studied numerically and theoretically, notably by Hunter (1990) Vakhnenko (1992), Parkes( 1993), Vakhnenko and Parkes (1998), Boyd (2004, 2005), Stepanyants (2006), Liu et al (2010) and Kraenkel et al (2011). We also note that it is readily shown that equation (4) does not have any smooth solitary wave solutions (see Liu et al (2010) and the Appendix of Grimshaw and Helfrich (2011), where the argument produced there still applies when $\lambda=0$ ), but does support a family of smooth periodic travelling wave solutions (Ostrovsky, 1978). Thus clearly not all solutions will break, although Boyd (2005) showed numerically that a large class of solutions will break, even when the amplitude is very small provided that the length scale is correspondingly also very small. However, it has been shown by Vakhnenko and Parkes (1998) that (4) is integrable, with soliton solutions, see also Vakhnenko (1992), Parkes( 1993) and Kraenkel et al (2011). This would seem to predict that breaking is prevented, contrary to the numerical results of Boyd (2005), and also to the proven non-existence of solitary waves. The purpose of this paper is to provide an explanation of these apparent contradictions.

We can rescale equation (4) by

$$
\tilde{x}=\gamma x, \quad \tilde{t}=t, \quad \tilde{u}=\mu \gamma u, \quad \tilde{v}=\mu \gamma^{2} v .
$$


Then equation (4) is obtained with $\mu=\gamma=1$, which we assume henceforth. In section 2 we examine the claimed integrability of (4) and show that although it is integrable there is also a very strong constraint on when integrability can hold. In section 3 we re-examine the soliton and periodic travelling wave solutions of (4) and demonstrate that the former violate the integrability constraint, while the integrability allows for an explicit determination of all the allowed periodic travelling waves. In section 4 we present a sample of our numerical results, which confirm those of Boyd (2005), and go further in indicating that when the integrability constraint fails, then breaking inevitably occurs. We conclude in section 5 with a summary and discussion.

\section{Integrability}

Vakhnenko and Parkes (1998) used a transformation of variables to establish integrability of (4), given by (but note the notation is changed here),

$$
x=\theta(X, T)=X+\int_{T_{0}}^{T} U\left(X, T^{\prime}\right) d T^{\prime}, \quad t=T, \quad u(x, t)=U(X, T) .
$$

Note that these are in fact characteristic co-ordinates, and can be specified by

$$
\frac{d x}{d t}=u, \quad \text { where } \quad x=X \quad \text { at } \quad t=T_{0} .
$$

It then follows that

$$
U_{T}=u_{t}+u u_{x}, \quad U_{X}=\phi u_{x}, \quad \phi=\theta_{X}=1+\int_{T_{0}}^{T} U_{X}\left(X, T^{\prime}\right) d T^{\prime}, \quad \phi_{T}=U_{X} .
$$

Thus equation (4) becomes

$$
U_{X T}=\phi U, \quad \text { and so } \quad U U_{X T T}-U_{T} U_{X T}=U^{2} U_{X} .
$$

Finally, putting

$$
U=V_{T}, \quad \text { where } \quad V=\int_{T_{0}}^{T} U\left(X, T^{\prime}\right) d T^{\prime}, \quad \text { and so } \quad \phi=1+V_{X},
$$

we see that (9) becomes

$$
V_{X T T}=\left(1+V_{X}\right) V_{T} .
$$

Note that the zero mass condition (3) becomes

$$
\int_{-\infty}^{\infty} V_{T}\left(1+V_{X}\right) d X=0
$$


Vakhnenko and Parkes (1998, 2002) have shown that (11), and hence (4), is integrable, and has loop soliton solutions constructed by the Hirota method. Note that they set $T_{0}=-\infty$. The Lax pair is

$$
\begin{aligned}
& \psi_{T T T}-V_{T} \psi_{T}-\Lambda \psi=0, \\
& 3 \psi_{X T}-\left(1+V_{X}\right) \psi-\nu \psi_{T}=0,
\end{aligned}
$$

where $\nu=\nu(X)$ is arbitrary, and the spectral eigenvalue $\Lambda$ is a constant. In this Lax pair formulation, $X$ is the "evolution" variable, and $T$ is the "spatial" variable, so that we cannot immediately use this Lax pair for an initial value problem. But importantly we note that although the integrability of (11) holds for all $X, T$, it does not necessarily hold for all $x, t$, as the implied integrability of the reduced Ostrovsky equation (4) will only then hold provided that the Jacobian of the transformation from $(x, t)$ to $(X, T)$ should not vanish. This Jacobian is $\phi$, and since $\phi=1$ at $T=T_{0}$, we infer that (4) is integrable provided that $\phi>0$. But if as $T=t \rightarrow t_{b}, \phi \rightarrow 0, \infty$, then the relations (8, 10) imply that $u_{x} \rightarrow-\infty$, and so breaking occurs.

In an apparently alternative approach, Kraenkel et al (2011) have shown that the original equation (4) in the $(x, t)$ coordinate system, also has a Lax pair, expressed in terms of a function $F$ where

$$
F^{3}=1-3 u_{x x} .
$$

In particular they show that $F$ is a conserved density,

$$
F_{t}+(u F)_{x}=0 .
$$

In the transformed variables this becomes

$$
(F \phi)_{T}=0, \quad \text { so that } F \phi=F_{0}(X) \text { where } F\left(X, T_{0}\right)=F_{0}(X),
$$

where we have used the boundary condition that $\phi=1$ at $T=T_{0}$, and we note that at $T=T_{0}, X=x$, so that $F_{0}(x)$ is determined by the initial conditions in the original variables. Further, we find that

$$
u_{x x}=\frac{1}{\phi}\left\{\frac{U_{X}}{\phi}\right\}_{X}=\frac{1}{\phi}\left\{\frac{\phi_{T}}{\phi}\right\}_{X}=\frac{1}{\phi}\left\{\frac{\phi_{X}}{\phi}\right\}_{T}=\frac{\phi \phi_{X T}-\phi_{X} \phi_{T}}{\phi^{3}}=\frac{\{\log \phi\}_{X T}}{\phi} .
$$

Indeed, the key result (15) can be established directly from (11) and (17). Next, we note that using (16) and the definition (14), the expression (17) takes either of the equivalent forms

$$
\begin{gathered}
(\log F)_{X T}=\frac{F_{0}}{3 F}\left(F^{3}-1\right), \\
\text { or }(\log \phi)_{X T}=\frac{\phi}{3}\left(1-\frac{F_{0}^{3}}{\phi^{3}}\right) .
\end{gathered}
$$

which are, respectively, equations for $F$ or $\phi$ alone. Then, as noted by Kraenkel et al (2011), (18) can be converted to the integrable Bullough-Dodd equation

$$
3 v_{Y T}=\exp (2 v)-\exp (-v), \quad \text { where } F=\exp (v), Y=\int_{X_{0}}^{X} F_{0}\left(X^{\prime}\right) d X^{\prime} .
$$


Note that transformations used by Vakhnenko and Parkes (1998, 2002) and that used by Kraenkel et al (2011) are equivalent, although apparently Kraenkel et al (2011) were unaware of this. The Lax pair of Kraenkel et al (2011) can now be obtained from that for the Bullough-Dodd equation (20) in the transformed variables $(X, T)$ variable with evolution in the $T=t$ variable; see also Faquir et al (2007) and the references therein, which indicate a connection with the Dym hierarchy, and presumably is equivalent to the Lax pair (13).

The transformation to the integrable Bullough-Dodd equation (20), requires that $F \neq 0$. In an infinite domain where all solutions are required to be localised, or in a periodic domain, there is always at least one point where $u_{x x}=0$, and hence at that point, $F=1$. We conclude that integrability holds when $F>0$, that is $3 u_{x x}<1$. The conservation law (16) then shows that this condition is completely equivalent to the condition that $\phi>0$. Indeed this condition also shows that as $\phi \rightarrow 0, \infty$, then $F \rightarrow \infty, 0$, and breaking occurs. We conclude that loss of integrability inevitably leads to breaking.

In more detail, we infer that if the initial condition $u(x, 0)=u_{0}(x)$ is such that $F(x, 0)=F_{0}(x)>0$ for all $x$, that is $3 u_{0 x x}<1$ for all $x$, then from (16) $0<F \phi=$ $F_{0}(X)<\infty$, integrability holds and there is no breaking. On the other hand, suppose that there is a set of intervals $x_{1} \leq x \leq x_{2}$ in which of which $F_{0}(x) \leq 0,3 u_{0 x x} \geq 1$, with equality only at the end points. Importantly, we observe that the value $F=0$ is conserved on characteristics, that is, if $F_{0}\left(X_{1,2}\right)=0$, then $F\left(X_{1,2}, T\right)=0, T \geq 0$ (note that now we set $T_{0}=0$ without loss of generality). Consequently when the initial value $F_{0}(X)$ takes both positive and negative values, then as long as the solution exists, that is $0<\phi<\infty$, the $X, T$ domain is divided into regions where $F<0$, namely the region between the characteristic boundaries $X=X_{1,2}$, and the remaining region where $F>0$. Formally, the reduction to (20) holds only when $F>0$. However, the conservation law (16) remains valid as long as $0<\phi<\infty$, and if $F<0$, (18) is replaced by

$$
3 F(\log [-F])_{X T}=F_{0}\left(F^{3}-1\right),
$$

while the Bullough-Dodd equation (20) is replaced by

$$
3 v_{Y T}=\exp (2 v)+\exp (-v), \quad \text { where } \quad-F=\exp (v), Y=\int_{X_{0}}^{X} F_{0}\left(X^{\prime}\right) d X^{\prime} .
$$

Formally (20) becomes (22) if $v$ is replaced $v+i \pi$, according to the change of sign of $F$, and hence we assume that (22) may also be integrable, albeit in the complex $v$-plane. However, the full equation is not integrable, with a breakdown on the lines $X=x_{1,2}$ where $F=0$.

Next, we note that equation (19) can be formally integrated once to yield

$$
\beta(X, T)=(\log \phi)_{X}=\int_{0}^{T} \frac{\phi}{3}\left(1-\frac{F_{0}^{3}}{\phi^{3}}\right) d T .
$$

where we have used the initial condition that $\phi=1$ at $T=0$. This holds in the interval $X_{1} \leq X \leq X_{2}$ where $F_{0} \leq 0$ (as well as in the remaining intervals where $F_{0}>0$ ), and 
then the integrand on the right-hand side is positive for all $\phi>0$, with a minimum value of $-2^{-2 / 3} F_{0}(X)$ achieved where $\phi=-2^{1 / 3} F_{0}(X)$, independently of $T$. Note that since $\phi=1$ at $T=0$, there always exists at least a finite domain in $T \geq 0$ where $\phi>0$. It follows that then $\beta>0$ in $X_{1} \leq X \leq X_{2}$, and so also $\phi_{X}>0$, with the consequence that $\phi$ cannot achieve a minimum value in this interval. Indeed, for each $X$ in the interval $X_{1}<X<X_{2}$

$$
\beta=(\log \phi)_{X}>-2^{-2 / 3} F_{0}(X) T,
$$

and integrating (24) over the interval $X_{1}<X<X_{2}$ yields

$$
\begin{gathered}
\phi\left(X_{1}, T\right)<\phi\left(X_{2}, T\right) \exp (-\alpha T), \\
\text { where } \alpha=2^{-2 / 3} \int_{X_{1}}^{X_{2}}\left(-F_{0}(X)\right) d X=2^{-2 / 3} \int_{X_{1}}^{X_{2}}\left\{3 u_{0 x x}(x)-1\right\}^{1 / 3} d x .
\end{gathered}
$$

Thus the Jacobian $\phi\left(X_{1}, T\right)$ at the left-hand end of the interval on which $F_{0}$ is negative becomes exponentially small compared to its value $\phi\left(X_{2}, T\right)$ at the right-hand end. Thus, provided the Jacobian $\phi\left(X_{2}, T\right)$ at the right-hand end does not grow exponentially fast, $\phi\left(X_{1}, T\right)$ at the left-hand end becomes arbitrarily small. This suggests that breaking will occur extremely close to the location $X=X_{1}$, but since $\phi_{X}\left(X=X_{1}, T\right)>0$ from the arguments described above, we infer that the first breaking event will occur just below $X=X_{1}$. In this vicinity, the slightest fluctuation will induce wave breaking. The bound (25) becomes more stringent the larger the area of negative $F_{0}$ in the interval $X_{1}<X<X_{2}$, with the e-folding time $1 / \alpha$ decreasing monotonically with increasing area.

\section{Travelling waves}

\subsection{Loop solitons}

Before describing our numerical results on breaking solutions, it is useful to consider the reduction of (4) for steady travelling waves. First we note that the the loop soliton solutions of (4) obtained by Vakhnenko and Parkes (1998) violate the integrability constraint that $\phi>0$, and hence that $3 u_{x x}<1$. In the $(X, T)$ variables the loop soliton solution is given by

$$
U=-\frac{3}{8 k^{2}} \operatorname{sech}^{2}(k \eta), \quad \eta=X-c T, \quad c=-\frac{1}{4 k^{2}} .
$$

In physical space, this loop soliton solutions (27) becomes

$$
u=-\frac{3}{8 k^{2}} \operatorname{sech}^{2}(k \eta), \quad \zeta=x+\frac{t}{4 k^{2}}=\eta-\frac{3}{2 k} \tanh (k \eta) .
$$

The condition $\phi>0$ becomes

$$
\phi=\theta_{X}=1-\frac{U}{c}=1-\frac{3}{2} \operatorname{sech}^{2}(k \eta)>0,
$$


which is clearly violated for all $\eta$ such that $\operatorname{sech}^{2}(k \eta)>2 / 3$. That is the transformation between $\zeta$ and $\eta$ is only valid for $\operatorname{sech}^{2} k \eta<2 / 3$. Also, the curvature (17) is

$$
u_{x x}=\frac{c^{2} U_{\eta}^{2}-U(c-U)^{2}}{(c-U)^{3}}=\frac{-U\left(c^{2}-c U+U^{2} / 3\right)}{(c-U)^{3}}, \quad \frac{U}{c}=\frac{3}{2} \operatorname{sech}^{2}(k \eta),
$$

which is negative for all $0<U / c<1$, but positive and greater than 3 for $1<U / c<3 / 2$, with a singularity at $U=c$. Thus the criterion that $u_{x x}<1 / 3$ is violated when $U=c$, precisely the same value when $\phi=0$.

\subsection{Periodic travelling waves}

The reduced Ostrovsky equation (4) has a family of periodic travelling waves, $u=$ $u(\zeta), \zeta=x-c t$ of period $2 L$, with a limiting wave with a parabolic shape and a corner crest, see Ostrovsky (1978), Grimshaw et al (1998), Boyd (2004) and Stepanyants (2006). This limiting wave is given by

$$
u=\frac{\zeta^{2}}{6}-\frac{c}{2}, \quad c=\frac{L^{2}}{9}, \quad-L<\zeta<L .
$$

Note that this has $3 u_{\zeta \zeta}=1$ and so is on the boundary of the allowed domain $3 u_{\zeta \zeta}<1$. The full family of periodic travelling waves can be obtained explicitly using a slight adjustment of the transformation (6) described above. Let $u=u(\zeta), \zeta=x-c t$, so that (4) becomes

$$
\left\{(u-c) u_{\zeta}\right\}_{\zeta}=u
$$

Now put

$$
\zeta=\eta-\frac{V(\eta)}{c}, \quad u(\zeta)=U(\eta)=V_{\eta}(\eta)
$$

so that (32) becomes

$$
c^{2} U_{\eta \eta}=U(U-c) .
$$

Note that if $(U, c)$ is a solution, then so also is $(U-c,-c)$. There are two families of periodic solutions, given by

$$
\begin{aligned}
U & =a \operatorname{cn}^{2}(k \eta ; m)+d \\
\text { where } \quad a & =-6 m k^{2} c^{2}, \quad \frac{c}{a}= \pm \frac{2\left(m^{2}-m+1\right)^{1 / 2}}{3 m} \\
\text { and } \frac{d}{a} & =\frac{\left[1-2 m \pm\left(m^{2}-m+1\right)^{1 / 2}\right]}{3 m} .
\end{aligned}
$$

Here $\mathrm{cn}^{2}(\cdot)$ is the Jacobi Elliptic function of modulus $m, 0<m<1$. The solution (35) has a period $2 K(\mathrm{~m}) / k$ in the variable $\eta$, and hence, on using the transformation (33) the corresponding period $2 L$ in the variable $\zeta$ is given by

$$
k L=\left[1-\frac{d}{c}-6 c k^{2}\left(1-m-\frac{E(m)}{K(m)}\right)\right] K(m),
$$


For the first family, as $m \rightarrow 1$, the solution (35) reduces to the solitary wave (27), and as $m \rightarrow 0$ it reduces to a linear sinusoidal wave with wavenumber $2 k$ in $\eta$-space, riding on a pedestal $d=c=-1 / 4 k^{2}$. However, the expression (38) then shows that $k L \rightarrow 0$ as $m \rightarrow 0$, and so with $L$ fixed, it follows that then $k \rightarrow 0$, and this limit is singular. For the second family, as $m \rightarrow 1$, the solution (35) reduces to a solitary wave riding on a pedestal $d=c=-2 a / 3=1 / 4 k^{2}$, and as $m \rightarrow 0$ it reduces to a linear sinusoidal wave with a wavenumber $2 k$ in $\eta$-space, riding on a zero pedestal with speed $c=1 / 4 k^{2}$. In this case, the expression (38) shows that $2 \pi k=L$ as $m \rightarrow 0$, and so $1 / 2 k$ is the wavenumber in $\zeta$-space. In physical space these solutions are given by

$$
\begin{aligned}
& u=a \operatorname{cn}^{2}(k \zeta ; m)+d, \quad \zeta=\eta-\frac{V(\eta)}{c}, \\
& \text { provided that } \quad \frac{\partial \zeta}{\partial \eta}=1-\frac{U(\eta)}{c} \neq 0,
\end{aligned}
$$

which is just the general condition $\phi>0$. Since this must hold for all $\eta$, and $a<0$, this condition will fail when $0 \leq d-c \leq-a$, that is, when

$$
0 \leq 2 m-1 \pm\left(m^{2}-m+1\right)^{1 / 2} \leq 3 m
$$

It is readily shown that the first family satisfies this condition for all $m, 0<m<1$, and hence cannot be physically realized. However, the second family does not satisfy this condition for any $m, 0<m<1$, and hence is physically realizable. Note that for this second family, it follows from $(33,38)$ that when $m=1, k L=3 / 2$, while

$$
u=\frac{1}{4 k^{2}}-\frac{3}{8 k^{2}} \operatorname{sech}^{2}(k \eta), \quad \zeta=\frac{3}{2 k} \tanh (k \eta),
$$

so that the parabolic limiting solution (31) is recovered. From this perspective, the limiting solution is a solitary wave! Also, the curvature is given by

$$
u_{x x}=\frac{c^{2} U_{\eta}^{2}-U(c-U)^{2}}{(c-U)^{3}}=\frac{1}{3}-\frac{D}{3(c-U)^{3}}, \quad D=(d-c)^{2}(c+2 d) .
$$

Since $D \geq 0, c-U \geq 0$ for all $m, 0 \leq m \leq 1$, it follows that $3 u_{x x} \leq 1$ for all $m$ with equality only at $m=1$.

\section{Numerical results}

It remains to reconcile the theoretical results of section 2 with the numerical calculations of Boyd (2005), and to this end some new simulations are reported here. Remarkably, Boyd showed numerically that for the initial condition $u(x, 0)=u_{0}(x)=b \cos k x$, the solutions break if $3 b k^{2}>1$, which is precisely the condition that $3 u_{0 x x}(x)>1$ for some $x$. However, this numerical result is limited to a sinusoidal initial wave, while the condition $3 u_{0 x x}(x)>1$ for some $x$, that is $\max \left[3 u_{0 x x}\right]>1$, is more general, and 


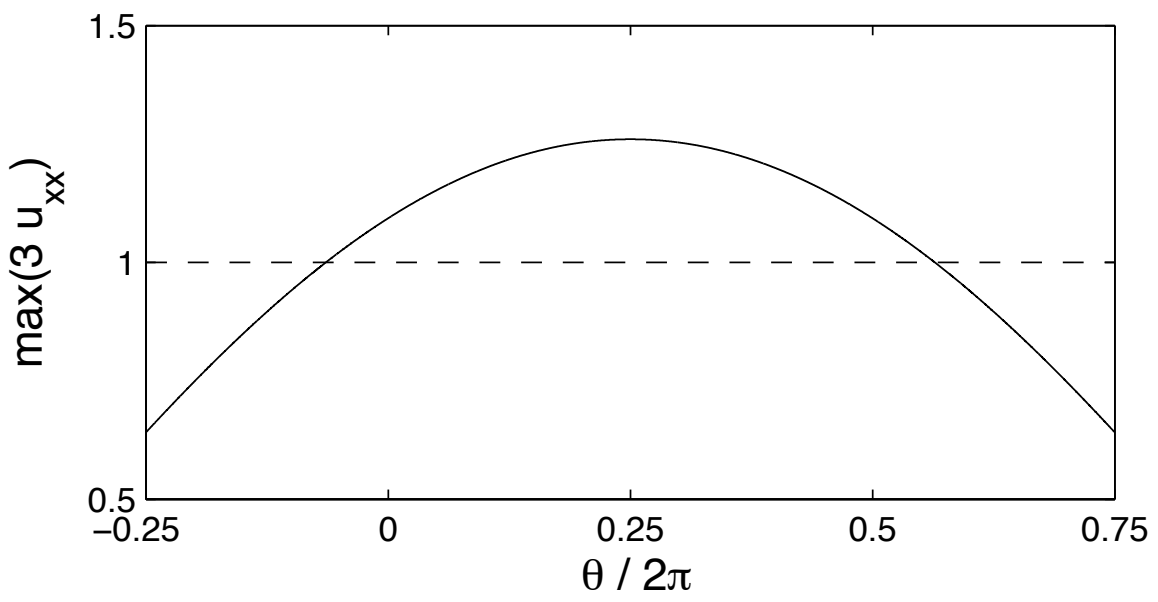

Figure 1: The maximum of $3 u_{0 x x}$ as a function of $\theta$ for the initial condition (43) with $u_{1}=0.3$ and $u_{2}=0.03$.

as discussed in section 2, points to the possibility that any initial state satisfying this condition will evolve to breaking. Indeed, Boyd presented one calculation with an initial condition composed of a superposition of two sinusoidal waves: one with $k=1$ and the second with $k=10$. The amplitudes of the individual harmonics were chosen so that the long wave satisfied $3 b k^{2}<1$ and the short wave had $3 b k^{2}>1$. In the calculation the long wave remained stable and smooth, while the short wave proceeded to breaking with 10 breaking crests. This seems to suggest that a general initial condition with a region where $3 u_{0 x x}>1$ will break. However, the significant amplitude and wave number separation in Boyd's two-wave example permitted the two waves to evolve essentially independently. Thus the breaking was interpreted solely as a consequence of the short wave exceeding the condition $3 b k^{2}>1$.

We further test the connection $\max \left[3 u_{0 x x}\right]>1$ to the occurrence of wave breaking, by solving the reduced Ostrovsky equation (4) (with $\mu=\gamma=1$ ) numerically. As Boyd (2005) notes, solutions of (4) on a domain of length $L$ can be rescaled to any other domain, thus our present calculations are for a periodic domain of length $2 \pi$. Two contrasting numerical schemes were used. The first was a Fourier-pseudospectral method with a fourth-order Runge-Kutta time integration for the equation in the original form (4). In all the calculations presented here the model was run with $N=4096$ grid points (or Fourier components). Anti-aliasing was applied to the calculation of the nonlinear term by zero-padding the Fourier transform to $2 N$ coefficients. The time step was varied between runs for numerical stability. This numerical model was tested by confirming $\mathrm{t}$ hat a single sinusoidal wave will break if $3 b k^{2} \geq 1.02$. The modest departure of the breaking condition from the theoretical value is consistent with Boyd's (2005) results and is due to the rapid increase in time to breaking as $3 b k^{2}$ approaches 1 from above and the accumulation of numerical errors. As in Boyd (2005), the occurrence of breaking was determined by monitoring the Fourier amplitudes of the numerical solution (see below). 

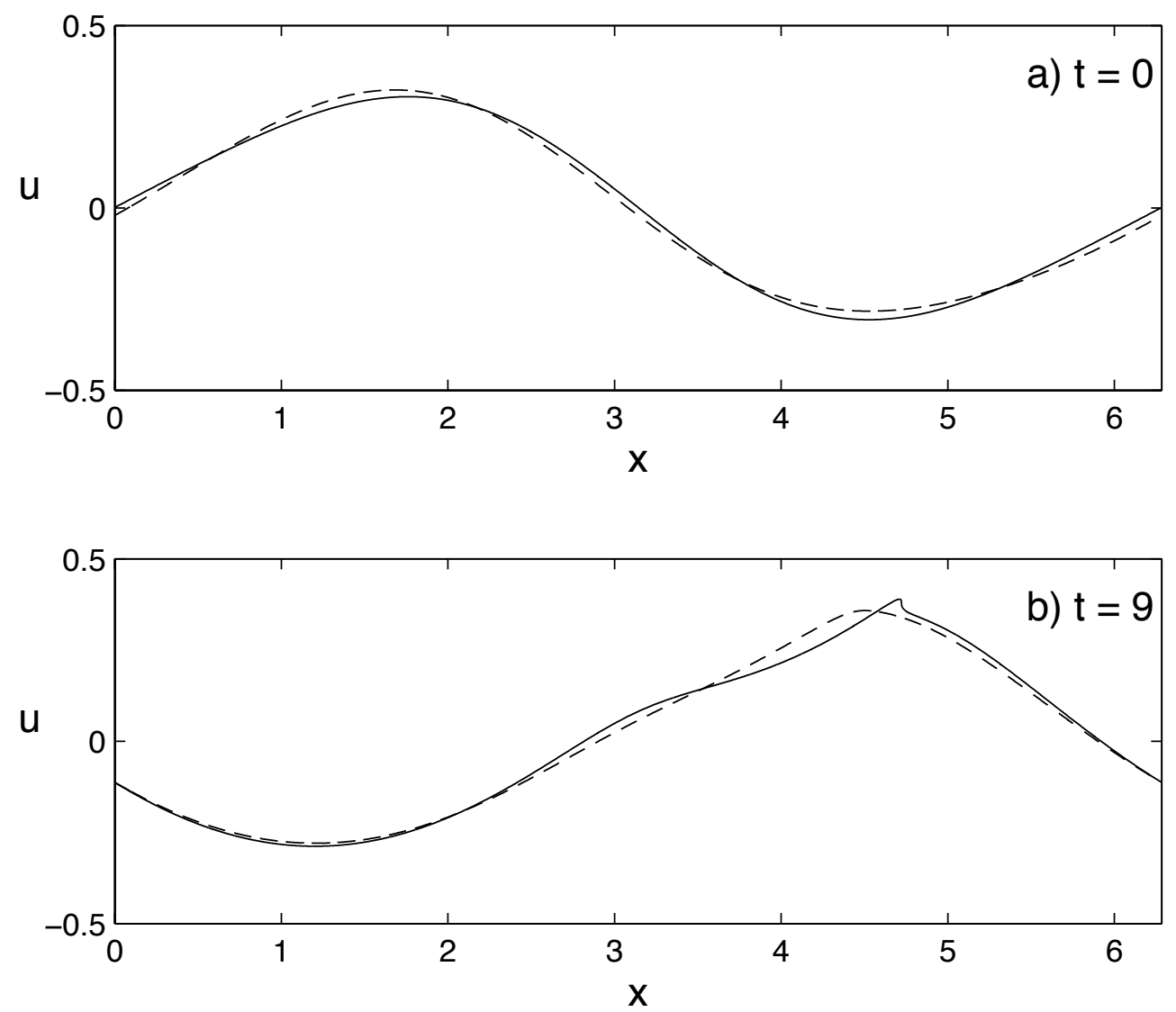

Figure 2: Numerical solutions of (4) with the initial condition (43) for $u_{1}=0.3$ and $u_{2}=0.03$ at $t=0$ (a) and $t=9$ (b). The dashed curves are for $\theta=3.913$ and $\max \left[3 u_{0 x x}\right]=0.9$ and the solid curves are for $\theta=3.109$ and $\max \left[3 u_{0 x x}\right]=1.1$. 

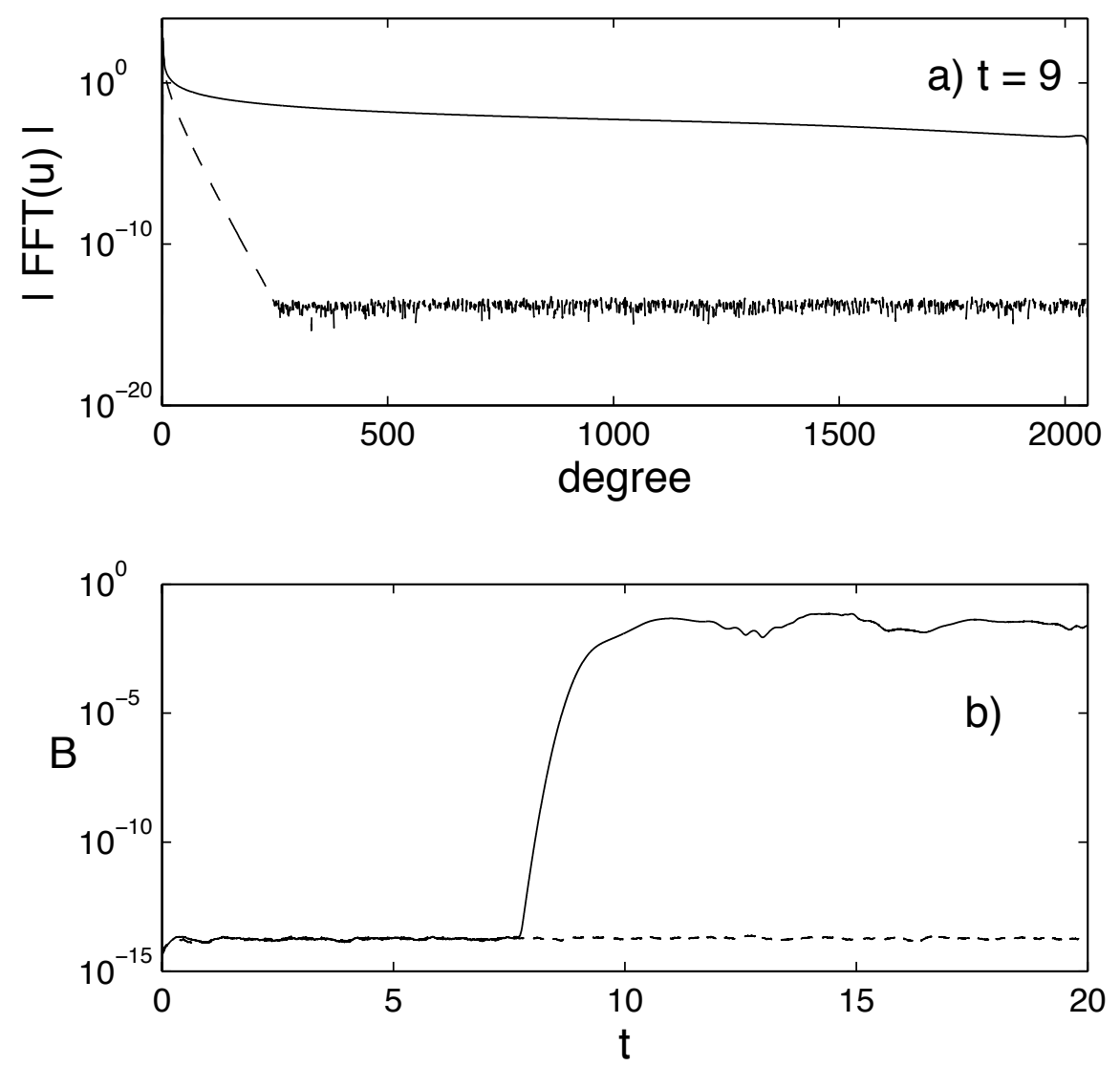

Figure 3: a) The magnitude of the Fourier coefficients at $t=9$ for the numerical solutions in Figure 2. b) Time series of $B$, the mean of the magnitudes of the Fourier coefficients of the highest 128 wave numbers. In both panels the solid lines are for $\max \left[3 u_{0 x x}\right]=1.1$ $(\theta=3.109)$ and the dashed lines for $\max \left[3 u_{0 x x}\right]=0.9(\theta=3.913)$. 


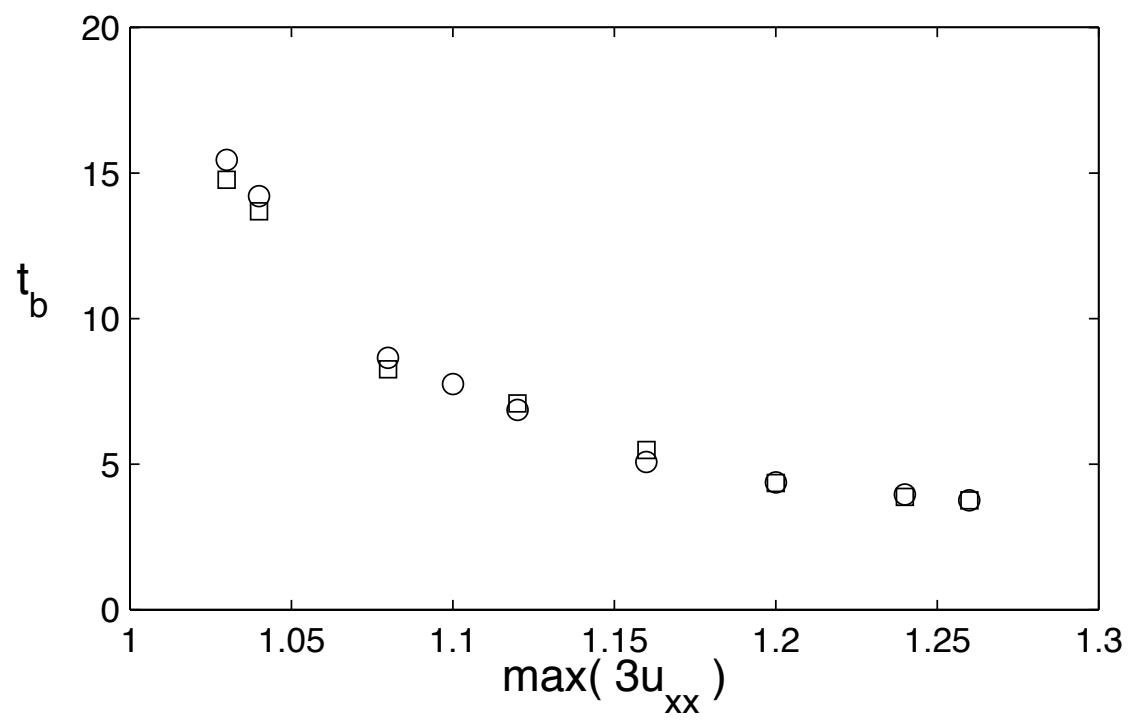

Figure 4: The time to breaking, $t_{b}$ versus $\max \left[3 u_{0 x x}\right]$ for the initial condition (43) with $u_{1}=0.3$ and $u_{2}=0.03$. The squares are for $-\pi / 2 \leq \theta \leq \pi / 2$ and the circles are for $\pi / 2 \leq \theta \leq 3 \pi / 2$

The second numerical method solves the reduced Ostrovsky equation in the characteristic form (9). Thus, let $W=U_{X}$ with inverse $U=\mathcal{I}(W)$ so that this characteristic form can be written as the first order pair,

$$
(\phi, W)_{T}=(W, \phi \mathcal{I}(W)),
$$

subject to the initial conditions that $\phi=1$ and $W=F_{0}^{\prime}(X)$ at $T=0$. Note that $U$ can be recovered from $W$ preserving the zero mean condition (3), by ensuring that the inverse operator $\mathcal{I}$ has the property that, for any $2 \pi$-periodic function $f(X)$,

$$
(\mathcal{I} f)_{X}=f \quad \text { and } \quad \int_{0}^{2 \pi} \mathcal{I} f d x=\int_{0}^{2 \pi}(\mathcal{I} f) \phi d X=0 .
$$

This system is solved to spectral accuracy for an unbounded interval in Esler et al (2009) (note that there is a misprint in their equation (26)) by expanding $\phi$ as a series of Chebyshev polynomials in $X$ with time-dependent coefficients. As noted there, solving in characteristic space is particularly useful for investigating wave-breaking as the wave breaks when the order unity quantity $\phi$ passes smoothly through zero. Esler et al (2009) show that characteristic integrations can be carried smoothly past breaking, where they agree closely with finite volume integrations which fit "equal area" shocks to the waves after breaking. The system (41) was integrated numerically with spectral accuracy by performing the integration in (42) in Fourier space and then normalising in real space using the result that the trapezium rule is spectrally accurate for periodic functions. Integrations up to $T=100$ with 2048 nodes showed that $F \phi$ was conserved 

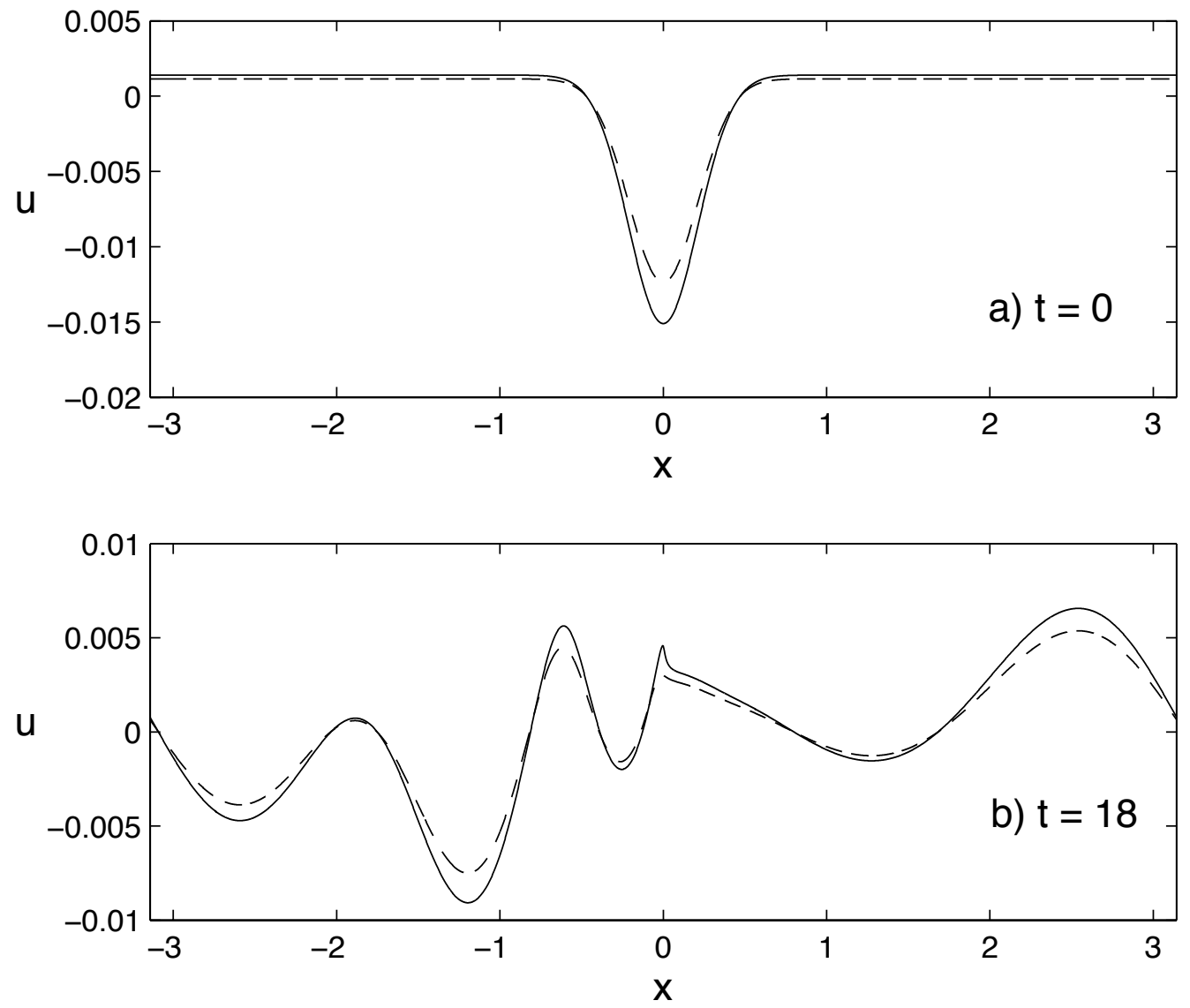

Figure 5: Numerical solutions of (4) with the initial condition (44) with $l=0.3$ and $b=-0.0165$ (solid lines) and $b=-0.0153$ (dashed lines) at $t=0$ (a) and $t=9$ (b). 


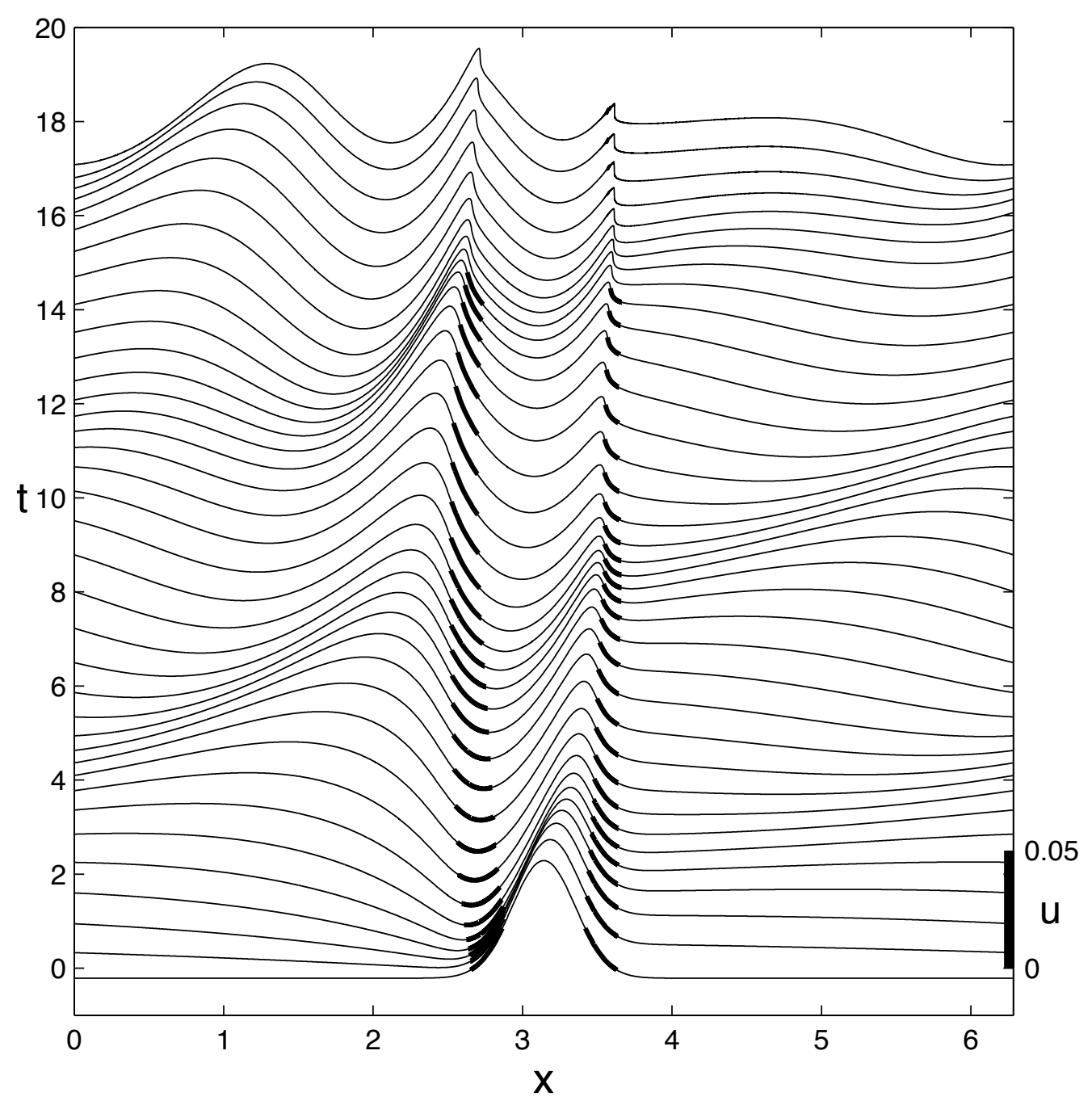

Figure 6: Numerical solution of (4) with the initial condition (44) with $l=0.3$ and $b=0.05$ for $0 \leq t \leq 18$ in intervals of 0.5 . The thick line segments indicate regions where $3 u_{x x}>1$ for $t<14$. 
with an accuracy of $10^{-8}$, and so the conservation of $F \phi$ (see (16)) was used explicitly by replacing (41) by the single equation (19). The advantage of this formulation is that (19) depends solely on the Jacobian $\phi$ which remains positive and of order unity until it vanishes when the wave breaks. The weakness of using (19) is that integrating past breaking is less straightforward than for the pair (41). Next we note that equation (19) can be formally integrated once, to yield (23). This equation was integrated for evenly-spaced nodes on $(0,2 \pi)$ using a Runge-Kutta method with $\phi$ obtained using the inverse operator $\mathcal{I}$, and normalised to spectral accuracy using the trapezium rule. This implementation proved extremely fast, accurate and robust.

\subsection{Fourier pseudo-spectral method results}

First, we present the results using the Fourier pseudo-spectral method. The initial condition is the superposition of a sinusoidal wave of length $2 \pi$ and its first harmonic,

$$
u_{0}(x)=u_{1} \sin (x)+u_{2} \sin (2 x+\theta),
$$

where $\theta$ is an arbitrary phase shift. The phase shift has two effects. The first is that the amplitudes $u_{1}$ and $u_{2}$ can be selected so that each individual component is subcritical, while varying $\theta$ causes the composite initial state to be either subcritical or supercritical to the criterion $\max \left[3 u_{0 x x}\right]>1$. This is illustrated in Figure 1 where max $\left[3 u_{0 x x}\right]$ from (43) is plotted as a function of $\theta$ for $u_{1}=0.3$ and $u_{2}=0.03$. Furthermore, for these values of $u_{1}$ and $u_{2}$ there is at most one region of $3 u_{0 x x}(x)>1$ in the domain.

Two initial waves from (43) with $u_{1}=0.3$ and $u_{2}=0.03$ are shown in Figure 2a. One (dashed) has $\theta=3.913$ and $\max \left(3 u_{x x}\right)=0.9$. The other (solid) has $\theta=3.109$ and $\max \left(3 u_{x x}\right)=1.1$. These two initial conditions are quite close to each other, yet only the case with $\max \left[3 u_{0 x x}\right]=1.1$ evolves to breaking. Figure $2 \mathrm{~b}$ shows the numerical solutions at $t=9$ and the supercritical initial condition has developed a small, but clear breaking front where $u_{x}$ exhibits a spike. The magnitudes of the Fourier components at $t=9$ for each wave are plotted in Figure 3a. The magnitudes of the components of the supercritical wave have increased and filled in for all Fourier degrees indicating breaking (Boyd, 2005). In contrast, the subcritical case exhibits the expected exponential fall-off with degree with a floor at the roundoff error limit. A good diagnostic for breaking is the average value, $B$, of the highest 128 degree Fourier coefficients. The time series of $B(t)$ for these two numerical runs is plotted in Figure $3 \mathrm{~b} . B(t)$ stays at the roundoff floor for the subcritical initial condition. For the supercritical initial condition, $B(t)$ increases rapidly at $t \approx 7.75$ and subsequently levels off. Note that the pseudospectral numerical solution of (4) is unreliable once the breaking has fully developed.

Additional numerical solutions for $u_{1}=0.3$ and $u_{2}=0.03$ with $\theta$ varied confirm that breaking emerges only for initial conditions with $\max \left[3 u_{x x}\right]>1.02$. Figure 4 shows the time to breaking, $t_{b}$, as a function of $\max \left[3 u_{0 x x}\right]$. Here $t_{b}$ is found from the intersection of two linear fits to $\log (B(t))$ on either side of the point of departure from the roundoff floor. The time to breaking increases rapidly as the critical condition is approached from above and decreases toward the non-rotating breaking time $t_{b}=1 / \max \left[-u_{0 x}\right]=$ 
$u_{1}^{-1}=3.33$ for the primary wave as $\max \left[3 u_{0 x x}\right]$ increases. The time to breaking is largely independent of the phase $\theta$ (i.e., details of the initial conditions). The squares and circles indicate $\theta \leq \pi / 2$ or $\theta \geq \pi / 2$, respectively (see Figure 1 ). Additional tests with different combinations of $u_{1}$ and $u_{2}$ give the same for the value of the breaking criterion and a similar behaviour for $t_{b}$ as a function of the supercriticality.

It is important to know whether the breaking criterion max $\left[3 u_{0 x x}\right]>1$ applies to all initial conditions. This is, of course, impossible to test numerically, but it is reasonable to consider an initial disturbance that is quite different from (43). Thus some additional calculations were undertaken with the Gaussian-shaped disturbance

$$
u(x, 0)=b \exp \left(-\frac{x^{2}}{l^{2}}\right)-b \frac{l}{2 \pi^{1 / 2}} \operatorname{erf}\left(\frac{\pi}{l}\right)
$$

in the periodic domain $-\pi \leq x \leq \pi$. Here $b$ is the amplitude and $l$ is the width scale. The second term in (44) is the domain average of first term so that the integral of $u(x, 0)$ over the domain is zero as required.

First consider the situation $b<0$. Then $\max \left[3 u_{x x}\right]=-6 b / l^{2}$ at $x=0$. Figure 5a shows two initial conditions for $l=0.3$ with $b=-0.0135$ and -0.0165 , so that $\max \left[3 u_{x x}\right]=0.9$ and 1.1 , respectively. The numerical solutions at $t=30$ are plotted in Figure 5. The supercritical, $b=-0.0165$, example has developed a breaking front near $x=0$. This is reflected in the $B(t)$ diagnostic (not shown) which indicates breaking occurs at $t_{b} \approx 24.8$. The subcritical case shows no evidence of breaking for $t \leq 100$.

When $b>0$ there are two regions where $\max \left[3 u_{x x}\right]>1$, centered at $x= \pm(3 / 2)^{1 / 2} l$, for $b>l^{2} e^{1.5} / 12$. A numerical solution with $l=0.3$ and $b=0.05$ such that $\max \left[3 u_{x x}\right]=$ 1.49 is shown in Figure 6. This example shows that the regions of $3 u_{x x}>1$ (indicated by the thick line segments for $t<14$ ) remain intact as the solution evolves and that breaking fronts emerge from each of these regions. For $t \geq 14$ additional scattered regions of $3 u_{x x}>1$ develop due to accumulation of energy at high wave numbers once the breaking begins $\left(t_{b} \approx 10.3\right)$ and are not shown since they are related to errors in

the numerical solution. Additional numerical experiments with (44) and variations of $b$ and $l$ again shown that breaking only emerges when the initial condition satisfies $\max \left[3 u_{0 x x}\right]>1.02$ for some range of $x$.

\subsection{Characteristic method results}

This numerical method has better accuracy, and is used here to refine the results obtained above. Let the minimum over $X$ at any time $T$ of the Jacobian occur at $X_{m}(T)$ and have value $\phi_{m}(T)=\phi\left(\left(X_{m}(T), T\right)\right.$. Figure $7\left(\right.$ a) shows $\log \left[\phi_{m}(T)\right]$ as a function of $T$ for the initial profile (43) with $u_{1}=0.3, u_{2}=0.03$ and $\theta_{0}=3.5453$ so that $\max \left[u_{0 x x}\right]-1 / 3=4 \times 10^{-5}$. The wave breaks when $\phi_{m}$ first vanishes, at $T=T_{b}=2081.7$ where $T_{b}$ is the time to breaking (note that in this subsection we consistently use $T$ instead of $t$ since we are now using characteristic variables, but of course $T=t$ are the same variable, see (6)). For this initial profile (26) gives $\alpha=6.21 \times 10^{-4}$ so $\alpha T_{b}=1.3$. Figure 7(a) includes a line of slope $\alpha$ showing that the decay of $\phi_{m}(T)$ is indeed bounded 
by (25) and is captured by (25) over the majority of the evolution. Figure 7(b) shows $\alpha T_{b}$ as a function of $\max \left[u_{0 x x}\right]-1 / 3$ for the same initial profile as in Figure 7 (a) but for varying $\theta_{0}$. Over two orders of magnitude change in the abscissa the ordinate varies by less than $5 \%$.

Figure 8 shows the Jacobian at the moment of breaking, $\phi\left(X, T_{b}\right)$, for the example of Figure $7(\mathrm{a})$ with Figure $8(\mathrm{a})$ showing one period in $X$ and Figure $8(\mathrm{~b})$ showing the detail of the wave in the region where $F_{0}(X)<0$. The scalings for Figure 8(b) follow from the discussion leading to (26) in section 2, but it is informative to derive them independently. Consider a weakly supercritical initial condition where $u_{0 x x}$ is smooth with a maximum at $X_{0}$ slightly exceeding $1 / 3$. Then, near $X=X_{0}$,

$$
u_{0 x x}=a-b\left(X-X_{0}\right)^{2}+\cdots,
$$

where $a=\max \left[u_{0 x x}\right]=u_{0 x x}\left(X_{0}\right)$ and $b=-(1 / 2) u_{0 x x x x}\left(X_{0}\right)>0$, and so

$$
\left[F_{0}(X)\right]^{3}=(3 a-1)\left[-1+\xi^{2}+\cdots\right]
$$

where $\xi=\left(X-X_{0}\right)[3 b /(3 a-1)]^{1 / 2}$ and $\xi= \pm 1$ corresponds to $X=X_{1}, X_{2}$ in the general case. The generic behaviour near the breaking location should be governed by a parameter-free version of (19) and so near breaking $\phi$ scales as $F_{0}$, exactly in accord with our analysis and the numerical integrations. Thus we write

$$
\phi=(3 a-1)^{1 / 3} \hat{\phi} .
$$

Then equation (19) becomes a parameter-free generic equation near breaking,

$$
(\log \hat{\phi})_{\xi \tau}=(\hat{\phi} / 3)\left[1+\left(1-\xi^{2}\right) / \hat{\phi}^{3}\right]
$$

where $T=\epsilon \tau$ for

$$
\epsilon=\frac{(3 a-1)^{5 / 6}}{(3 b)^{1 / 2}} .
$$

As expected, (49) differs from the general expression (26), applied in this limit, by only a multiplicative constant. As can be seen in Figure $8(\mathrm{~b})$, the form (46) means that $F_{0}$ has an infinite gradient at $X=X_{1,2}$. This is the generic behaviour for a smooth function $u_{0}(x)$ but there is no such restriction on (19) nor in the analysis leading to (25) and (26), and also no restriction there that $u_{0 x x}$ should only slightly exceed $1 / 3$. As $3 u_{0 x x} \rightarrow 1+$ in Figure $7(\mathrm{~b}), \alpha T_{b}$ approaches a non-zero value, corresponding to $\tau=\tau_{b} \sim 1$.3. This value could be found directly from integrating (48) subject to an arbitrary admissible initial condition and appropriate boundary conditions, which appear to be that $\phi / F_{0}=O 1$ as $\xi \rightarrow \pm \infty$.

Figure 8(b) shows that the Jacobian first vanishes when $X_{m}$ is indistinguishably close to $\xi \rightarrow-1$ from below, that is as $X \rightarrow X_{1}$ from below. Since $X_{m}$ is also a minimum of $\log \phi$,

$$
\beta\left(X_{m}(T), T\right)=0, \quad \text { and } \quad \beta_{X}\left(X_{m}(T), T\right)>0
$$


Differentiating with respect to $T$ and using (23) gives

$$
\beta_{X} \dot{X}_{m}+(1 / 3) \phi_{m}\left[1-\left(F_{0}\left(X_{m}\right) / \phi_{m}\right)^{3}\right]=0 .
$$

Once $\phi_{m}<F_{0}$, and this always occurs for sufficiently long integrations given the shapes of $\phi$ and $F_{0},(51)$ shows that $\dot{X}_{m}>0$. That is, the minimum of the Jacobian moves monotonically to the right. Figure 9 shows $\log _{10}\left[X_{1}-X_{m}(T)\right]$ for the same initial profile as in Figure 7(a). The distance decreases slightly faster than the minimum of the Jacobian showing that breaking for weakly supercritical initial conditions first occurs at the point corresponding to the left-hand edge of the region where $u_{0 x x}$ exceeds $1 / 3$.

Figure 10 shows the minimum of the Jacobian, $\phi_{m}(T)$, as a function of time for the profile of Figure $7(\mathrm{a})$, and for $T>400$. The dashed line shows the corresponding value of $F_{0}$ at the same $X$ and $T$, i.e. $F_{m}(T)=F_{0}\left(X_{m}(T)\right)$. On average $\phi_{m}$ is less than $\frac{1}{2} F_{m}$ and so the second term on the right-hand side of (19) is approximately 8 times the first. The nearer to breaking the greater is this disparity. Thus breaking is governed in general by the approximate equation

$$
(\log \phi)_{X T}=-\frac{F_{0}^{3}}{3 \phi^{2}},
$$

with $F_{0}^{3}=2(-1+\xi)$ in the local equation (48). Equation (52) has the solution

$$
\phi=A+B(X) T
$$

for $A$ constant and $B(X)$ a function of $X$ alone, provided $A B_{X}(X)=-F_{0}^{3} / 3$. Thus near breaking

$$
\phi=A\left(1-\frac{T}{T_{b}}\right)+\frac{T}{3 A} \int_{X}^{X_{1}} F_{0}^{3}\left(X^{\prime}\right) X^{\prime} .
$$

Since $F_{0}>0$ in $X<X_{1}$ and $F_{0}<0$ in $X>X_{1}$ the expression (54) shows that $\phi$ is increasing monotonically with distance from a local minimum at $X=X_{1}$ of

$$
\phi_{m}=A\left(1-\frac{T}{T_{b}}\right)
$$

For the local equation (48) this becomes

$$
\hat{\phi}=A\left(1-\tau / \tau_{b}\right)+(2 \tau / 3 A)(\xi+1)^{2},
$$

with $\hat{\phi}_{m}=A\left(1-\tau / \tau_{b}\right)$. The form (55) is consistent with Figure 10 where the minimum of the Jacobian does indeed appear to decrease linearly with $T$ until vanishing at $T_{b}$.

\section{Conclusion}

Our numerical results and analysis for the reduced Ostrovsky equation (4), together with the numerical results of Boyd (2005), strongly point to several conclusions regarding 


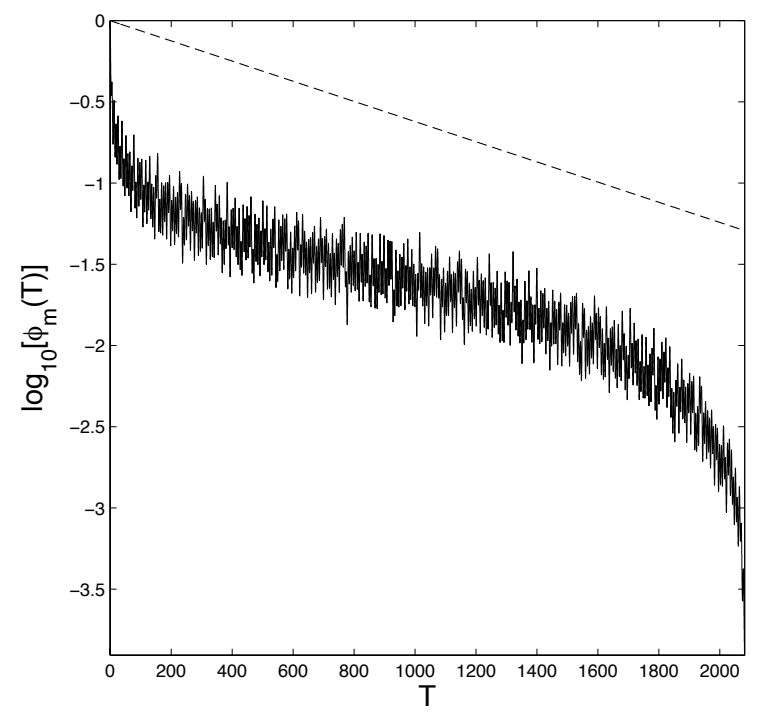

(a)

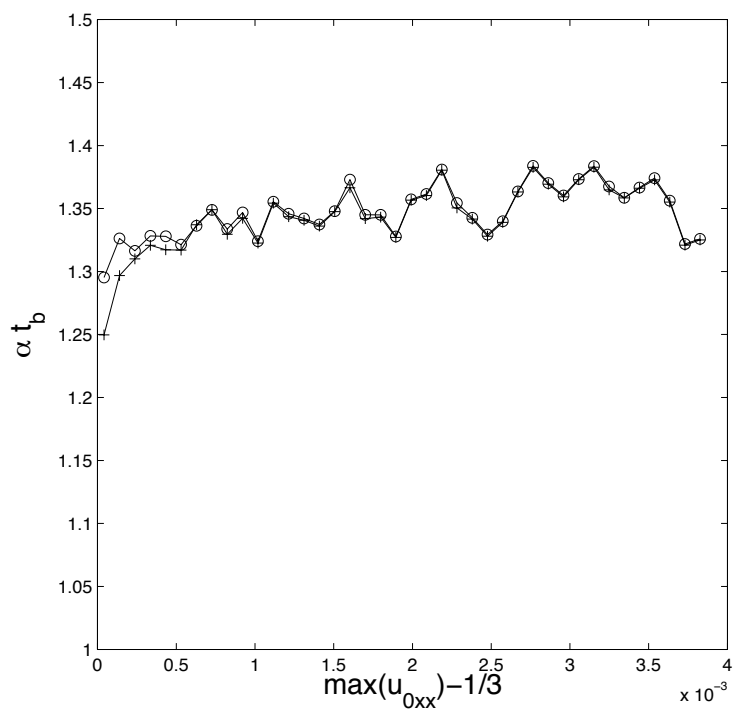

(b)

Figure 7: (a) The logarithm of the minimum, $\phi_{m}(T)$, over $X$ of the Jacobian $\phi(X, T)$ as a function of $T$ for the initial profile (37) with $u_{1}=0.3, u_{2}=0.03$ and $\theta_{0}=3.5453$ so $\max \left[u_{0 x x}\right]-1 / 3=4 \times 10^{-5}$, computed with $N=4096$ nodes. The wave breaks when $\phi_{m}$ first vanishes, at $T=T_{b}=2081.7$. (b) The scaled time to breaking, $\alpha T_{b}$, for this initial profile for varying $\theta_{0}$ as a function of the excess of $u_{0 x x}$ over $1 / 3$. The number of nodes in the computations are: ' + ' $N=2048$ and 'o' $N=4096$. 


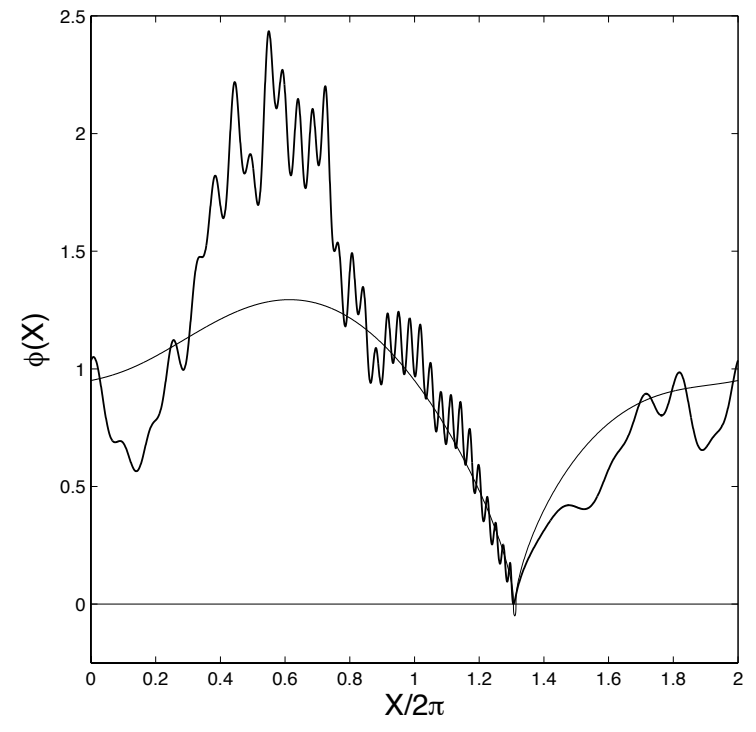

(a)

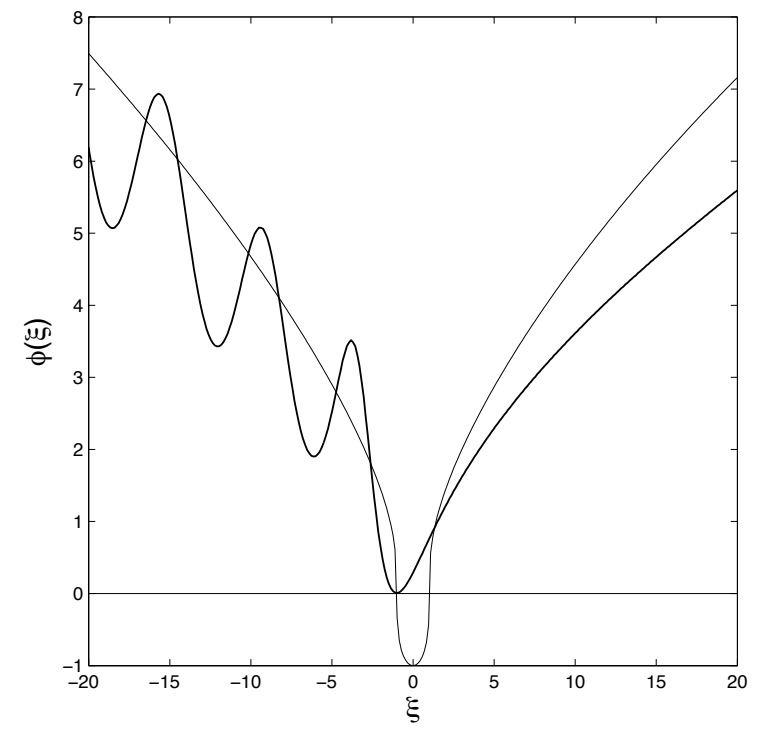

(b)

Figure 8: (a) The Jacobian $\phi\left(X, T_{b}\right)$ at the instant of breaking for the evolution of Figure 7. The thinner curve shows $F_{0}(X)$ which is negative in a region surrounding $1.31 \pi$. (b) The scaled Jacobian $\hat{\phi}(\xi)$ as a function of the scaled co-ordinate $\xi$. The scaling is such that the region of negative $F_{0}(X)$ has unit depth and width 2 .

the breaking, or non-integrability, criterion. In every case where the initial condition $u(x, 0)=u_{0}(x)$ is such that the integrability criterion is violated, that is,

$$
3 u_{0 x x}(x)>1 \text { for some } x
$$

breaking occurred. This suggests that solutions that begin in a supercritical region cannot evolve to a subcritical state without breaking. Further, every localized region where (57) is satisfied produces a separate breaking event. We infer that the solutions of the reduced Ostrovsky equation (4) belong to one of two classes. Either $3 u_{0 x x}(x)<1$ for all $x$ and then the equation is integrable and the solution exists for all time. Or this criterion is violated locally, and then breaking always occurs. Of course, our numerical and analytical results combined with the integrability criterion, that is $3 u_{x x}<1$ for all $(x, t)$, do not provide a complete rigorous proof of this statement, but the evidence we have presented seems compelling. Boyd (2005) pointed out that the transformation $u(x, t)=D^{2} u(\tilde{x}, \tilde{t})$ where $\tilde{u}=D^{2} u, \tilde{x}=x / D, \tilde{t}=D t$ leaves equation (4) unchanged for all values of $D \neq 0$. Significantly $u_{x x}=u_{\tilde{x} \tilde{x}}$ and so the breaking condition (57) is independent of this scaling transformation.

We note that Hunter (1990) showed that an initial condition for (4) on a periodic domain, that has a sufficiently steep negative slope will lead to breaking. A similar conclusion was also reached for the infinite line by Rosales and Grimshaw (private communication, see Boyd (2005)). Recently Liu et al (2010) obtained similar results with improved conditions for breaking to occur. Specifically, Hunter (1990) set the period 


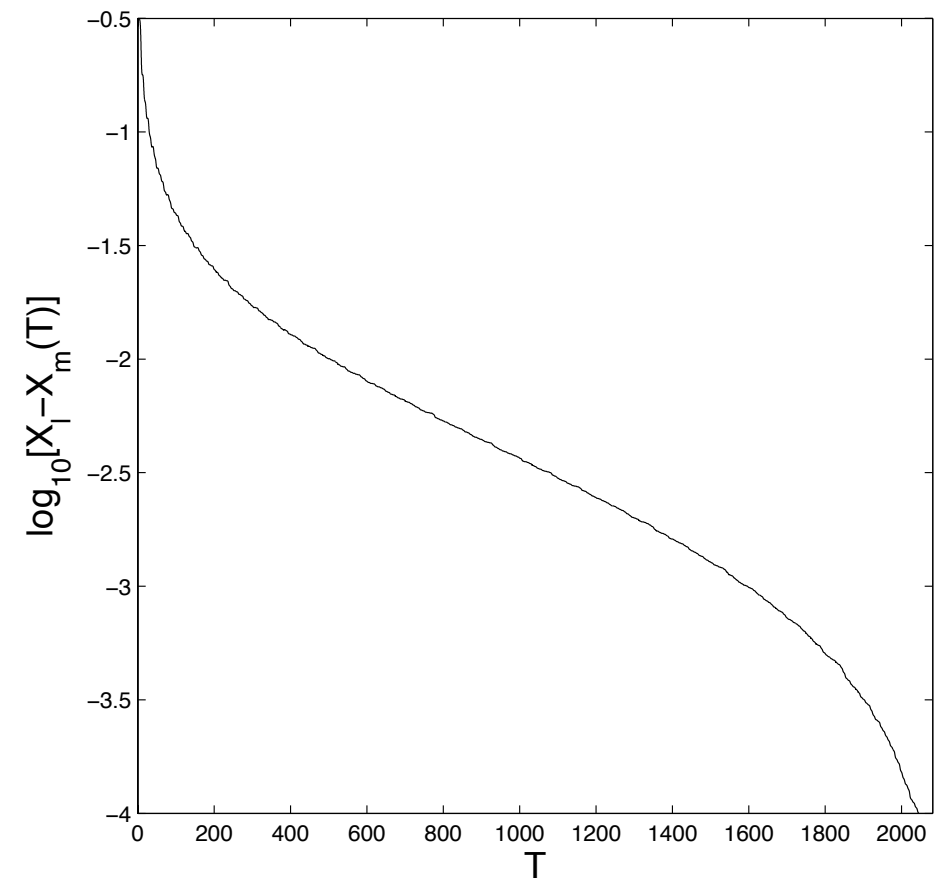

Figure 9: The logarithm of the distance that the position $X_{m}(T)$ of the minimum of the Jacobian lies below the left edge $X_{1}$ of the interval where $F_{0}<0$. The parameters are the same as in Figure 7(a). 


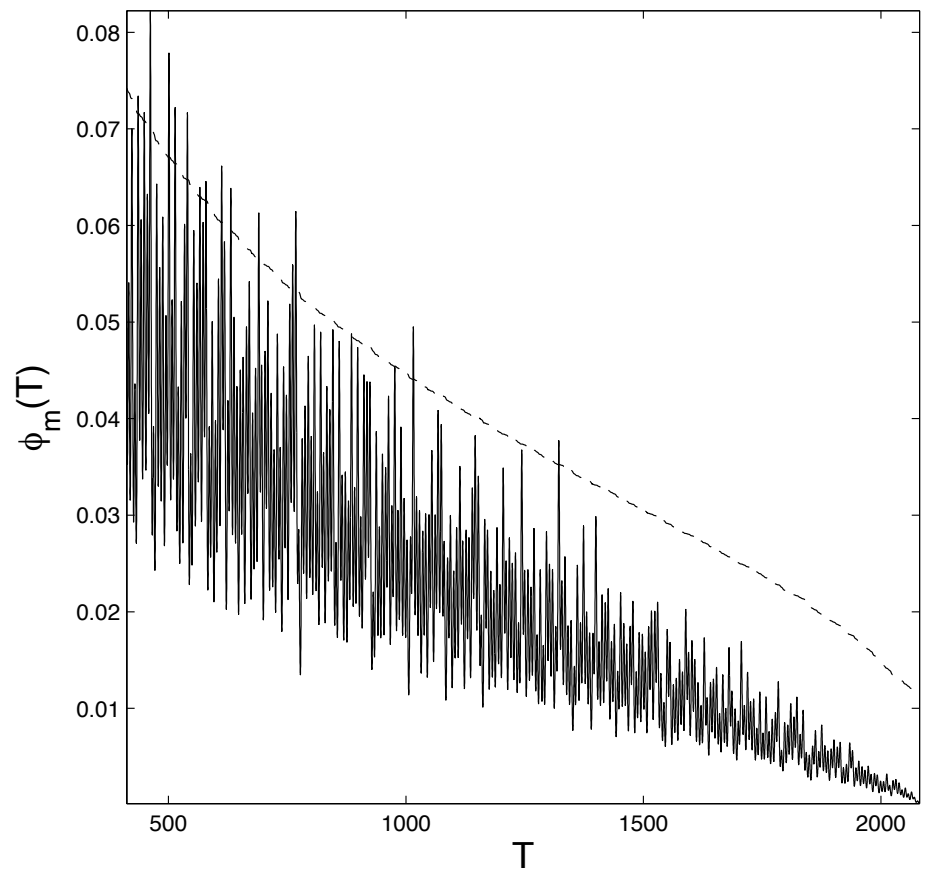

Figure 10: The minimum of the Jacobian, $\phi_{m}(T)$, as a function of time for $T>400$. The dashed line shows the corresponding value of $F_{0}$ at the same $X$ and $T$, i.e. $F_{m}(T)=$ $F_{0}\left(X_{m}(T)\right)$. The parameters and initial profile are the same as in Figure $7($ a). 
$2 L=1$ and examined that characteristic emerging from that initial point $X=x_{m}$ where the initial condition $u_{0}(x)$ has a maximum negative slope, $u_{0 x}(x) \geq u_{0 x}\left(x_{m}\right)=-m$, and deduced that if $m^{3}>4 M(m+4)$ where $M=\max \left|u_{0}\right|$, then breaking occurs. But at this location $u_{0 x x}\left(x_{m}\right)=0$, and so it lies within the region where the initial value of $F=F_{0}(x)>0$. From our discussion of integrability in section 2 and our numerical results in section 4 , we expect breaking to occur only at the edge of this region. Alternative breaking criteria were obtained by Liu et al (2010), who used a priori estimates to deduce that breaking occurs when either $J>(3 H / 2)^{3 / 2}$ or when $J>0, H>3 / 4$, where $J=-\int_{I}\left(u_{0 x}\right)^{3} d x, H=\left(\int_{I}\left|u_{0}\right|^{2} d x\right)^{1 / 2}$ and $I$ is again the periodic domain with period $2 L=1$. They also obtained an analogous criterion on the infinite line. However, we note that all these criteria can take the form $m^{2}>C M$ for some constant $C>0$. In the Appendix we show that if $m^{2}>4 M / 3$, see (61), then our breaking criterion (57) holds, whereas $C=4$ for Hunter (1990) and $C=3 / 2$ for Liu et al (2010). Noting that (61) is only a sufficient condition for breaking, we infer that (57) is, in general, a substantial improvement, and indeed, we contend is the optimal breaking criterion. We note that Boyd's initial condition, $u_{0}(x)=b \cos k x$, leads to $M=b, m=k b$ and the criterion (57) becomes $3 b k^{2}>1$, that is $3 m^{2}>M$ and so then $C=1 / 3$. Further, in all the numerical examples we have displayed here where breaking occurred, while the criterion (57) was satisfied, the criterion of Hunter (1990) was not satisfied. The criteria obtained by Liu et al (2010) are improvements of Hunter's criterion, but as they found in some numerical simulations, are far from optimal. We have confirmed that in all cases where they found breaking (see their Figures 2,3,4) but their breaking criteria were not satisfied, our breaking criterion (57) is satisfied.

It is pertinent to note that the breaking front first appears at a location in $x$ at the left-hand edge of the domain where $3 u_{0 x x}>1$ in the initial condition. The "local" disturbance that evolves to the breaking front originates in the region where $3 u_{0 x x}>1$ and then travels to the breaking location. In our simulations, where $3 u_{0 x x}$ only marginally exceeds unity, the travel speed is close to zero. This is readily apparent in the contour plot in Figure 6, and in the detailed plots of the Jacobian in Figures 7 and 9. Note that in the fixed reference frame, this corresponds to travelling at the linear long wave speed, see (2) and the subsequent discussion. This is a consequence of the fact that most simulations we have done have $3 u_{0 x x}$ only marginally greater than 1 , but nonetheless is a predicted general result, which has implications for where solitary-like waves will first appear when the $\lambda u_{x x x}$ term in the full Ostrovsky equation (2) is restored. That is, we expect that such solitary waves will appear first at the left-hand margins of these localised supercritical zones. When we revert to the original coordinates through the transformation (5), the breaking criterion becomes

$$
3 \mu u_{0 x x}(x)>\gamma, \quad \text { for some } x \text {. }
$$

Note that breaking is then enhanced when nonlinearity is increased, that is as $|\mu|$ is increased, but is suppressed as rotation is increased, that is $\gamma$ is increased.

Although this study is based entirely on the canonical model equation (4), it is useful to extrapolate the implication of the obtained results to the possible appearance 
of internal solitary waves in the coastal ocean following the generation of an internal tide. In the absence of rotation, there is an expectation based on the Korteweg-de Vries equation model, that internal solitary waves will inevitably form as the long-time outcome. However, as discussed by Helfrich (2007), Grimshaw and Helfrich (2008) and Helfrich and Grimshaw (2008) the effect of rotation is to inhibit this process. As noted above the breaking criterion (58) can be used to infer whether or not internal solitary-like waves, with much shorter length scales, will appear. Indeed, Farmer et al (2009) and Li and Farmer (2011) successfully used this condition in a study of large amplitude internal solitary waves in the South China Sea. They converted Boyd's (2005) breaking criterion for the special sinusoidal initial condition, which we have established is in fact universal, to a so-called Ostrovsky number, which was used to characterize the respective roles of nonlinearity and dispersion. Adapting their approach, we define the Ostrovsky number as

$$
O_{s}=\frac{3 \mu \kappa}{\gamma}, \quad \text { where } \quad \kappa=\max \left[u_{0 x x}(x)\right]
$$

Thus $\kappa$ is the maximum curvature in the initial condition, and for a localised initial pulse of amplitude $A$ and length scale $k^{-1}$, we can estimate that $\kappa \sim A k^{2}$. Thus when $O_{s}>1$, we expect to see localized "breaking" and the formation of internal solitary-like waves. But if $O_{s}<1$ we expect that no such "breaking" will occur, and consequently the formation of internal solitary-like waves is inhibited. Although we have defined $O_{s}$ in terms of the initial data, we note that in practice the initial time can be chosen to be any suitable time during the initial generation process of the internal tide, when the system is still controlled by linear wave dynamics.

\section{Acknowledgments}

KRH was supported by grant N00014-09-10227 from the Office of Naval Research.

\section{References}

[1] Boyd, J.P. (2004) Ostrovsky and Hunter's generic wave equation for weakly dispersive waves: matched asymptotic and pseudospectral study of the paraboloidal travelling waves (corner and near-corner waves), Euro. J. Appl. Maths., 16, 65-81.

[2] Boyd, J. P. (2005) Microbreaking and polycnoidal waves in the Ostrovsky-Hunter equation, Physics Letters A, 338, 36-43.

[3] Esler, J. G., Rump, O. J. and Johnson, E.R. (2009). Supercritical rotating flow over topography. Phys. Fluids 21, 066601.

[4] Faquir, M., Manna, M.A. and Neveu, A. (2007) An integrable equation governing short waves in a long-wave model, Proc. Roy. Soc., 463, 1939-1954. 
[5] Farmer, D., Li, Qiang and Park, Jae-Hun (2009) Internal wave observations in the South China Sea: the role of rotation and non-linearity, Atmosphere-Ocean, 209, 267-180.

[6] Galkin, V. N. and Stepanyants, Yu. A. (1991) On the existence of stationary solitary waves in a rotating fluid. J. Appl. Maths. Mech., 55, 939-943.

[7] Grimshaw, R. (1985). Evolution equations for weakly nonlinear, long internal waves in a rotating fluid. Stud. Appl. Math., 73, 1-33.

[8] Grimshaw, R. (2001). Internal solitary waves. Environmental Stratified Flows, ed. R. Grimshaw, Kluwer, Boston, Chapter 1, 1-29.

[9] Grimshaw, R. and Helfrich, K.R. (2008). Long-time solutions of the Ostrovsky equation. Stud. Appl. Math., 121, 71-88.

[10] Grimshaw, R. and Helfrich, K.R. (2011) The effect of rotation on internal solitary waves, IMA J. Appl. Math., (submitted).

[11] Grimshaw, R. H. J., Ostrovsky, L. A., Shrira, V. I. and Stepanyants, Yu. A. (1998) Long nonlinear surface and internal gravity waves in a rotating ocean, Surveys Geophysics, 19, 289-338.

[12] Helfrich, K. R. (2007) Decay and return of internal solitary waves with rotation, Phys. Fluids, 19, 026601 (9 pages).

[13] Helfrich, K. R. and W. K. Melville, W.K. (2006) Long nonlinear internal waves, Ann. Rev. Fluid Mech., 38, 395-425.

[14] Helfrich, K. R. and Grimshaw, R.H.J. (2008). Nonlinear disintegration of the internal tide, J. Phys. Ocean., 38, 686-701.

[15] Hunter, J. K. (1990) Numerical solution of some nonlinear dispersive wave equations, Computational Solution of Nonlinear Systems of Equations, ed. E. L. Allgower and K. Georg, Lectures in Applied Mathematics, 26, 301-316.

[16] Kraenkel, R., Leblond, H. and Manna, M. A. (2011) An integrable evolution equation for surface waves in deep water, arXiv:1101.5773v1.

[17] Leonov, A. I., (1981) The effect of Earth rotation on the propagation of weak nonlinear surface and internal long oceanic waves, Annals New York Acad. Sci., 373, 150-159.

[18] Li, Qiang and Farmer, David M. (2011) The generation and evolution of nonlinear internal waves in the deep basin of the South China Sea, J. Phys. Ocean., 41, 1345-1363. 
[19] Liu, Yue, Pelinovsky, Dmitri and Sakovich, Anton (2010) Wave breaking in the Ostrovsky-Hunter equation, SIAM J. Math.Anal., 42, 1967-1985

[20] Ostrovsky, L. A. (1978) Nonlinear internal waves a in rotating ocean, Oceanology, 18, 119-125.

[21] Parkes, E. J. (1993) The stability of solutions of Vakhnenkos equation, J. Phys. A Malh. Gen., 26, 6469-6475.

[22] Stepanyants, Y. A. (2006) On stationary solutions of the reduced Ostrovsky equation: Periodic waves, compactons and compound solitons, Chaos, Solitons and Fractals, 28, 193-204.

[23] Vakhnenko, V. A. (1992) Solitons in a nonlinear model medium, J. Phys. A: Math. Gen., 25, 4181-4187.

[24] Vakhnenko, V. O. and Parkes, E. J. (1998) The two loop soliton solution of the Vakhnenko equation, Nonlinearity, 11, 1457-1464.

[25] Vakhnenko, V. O. and Parkes, E. J. (2002) The calculation of multi-soliton solutions of the Vakhnenko equation by the inverse scattering method, Chaos, Solitons and Fractals, 13 1819-1826

\section{Appendix}

Consider the initial value $u_{0}(x)=u(x, 0)$. Each of $u_{0}, u_{0 x}, u_{0 x x}$ are either bounded periodic functions with period $2 L$, or localized bounded functions on the infinite line. Since each has a zero mean, each must take both positive and negative values. Suppose that $\tilde{m}^{2}=\max \left[u_{0 x}^{2}\right]$ and $M_{ \pm}=\max \left[ \pm u_{0}\right]$. Then in the phase plane $\xi=u_{0}, \eta=u_{0 x}^{2}$ since the function $u_{0}(x)$ generates a closed periodic curve which reaches all boundaries in the domain $M_{-} \leq \xi \leq M_{+}, 0 \leq \eta \leq \tilde{m}^{2}$, it is elementary that

$$
\begin{aligned}
& \frac{d \eta}{d \xi}>\frac{\tilde{m}^{2}}{R}, \quad R=M_{+}-M_{-}, \quad \text { somewhere, } \\
& \text { and so } \quad u_{0 x x}>\frac{\tilde{m}^{2}}{2 R} \text { for some } x .
\end{aligned}
$$

If $M=\max \left|u_{0}\right|$, then $R \leq 2 M$, and we also note that $\tilde{m} \geq m=\max \left[-u_{0 x}\right]$. Hence we infer that,

$$
\text { if } m^{2}>\frac{4 M}{3} \text {, then } u_{0 x x}>\frac{1}{3} \text { for some } x .
$$

This then provides a sufficient condition for breaking to occur, but as the slope estimate in (60) is not optimal, being essentially the simplest possible, it is not also a necessary condition. We note that for the limiting periodic wave $(31), m^{2}=M$, and so, in this case (61) is quite close to optimal. But for the initial condition $u_{0}=b \cos k x$ used by Boyd (2005), breaking occurred when $m^{2}>M / 3$, and then (61) is far from optimal. 